\title{
Derechos Humanos y Trabajo Social en México: nociones, compromisos y vocación en el estudiantado
}

Human rights and Social Work in Mexico: notions, commitments and vocation in the student

\section{Luis M. Rodríguez Otero}

Doctor en Menores en situación de desprotección y conflicto social.

Facultad de Trabajo Social de Culiacán

Universidad Autónoma de Sinaloa (México)

Miembro del Sistema Nacional de Investigadores

E-mail: luismaotero@yahoo.es

\section{Elisa Cerros Rodríguez}

Doctora en Filosofía con orientación en Trabajo Social y políticas comparadas de Bienestar Social.

Departamento de Trabajo Social

Universidad de Guadalajara (México)

Miembro del Sistema Nacional de Investigadores

E-mail: elisacerros@yahoo.com.mx 
Resumen: Los Derechos Humanos representan uno de los principios fundamentales en la definición internacional del Trabajo Social. Se plantea una investigación cuantitativa mediante un diseño descriptivo-correlacional con el objetivo de analizar las actitudes del alumnado de la licenciatura de Trabajo Social mexicano hacia los derechos humanos. Se aplicó un cuestionario autoadministrado a 610 alumnos/as de la Universidad Autónoma de Nuevo León (UANL) y la Universidad de Guadalajara (UdeG), seleccionados a través de un muestreo aleatorio estratificado. En el instrumento se incluyeron: (1) un cuestionario sociodemográfico, (2) la escala de componente vocacional, (3) la escala de ámbitos de intervención de Trabajadores/as Sociales, (4) la escala Likert de resiliencia y (5) la Escala de Nociones sobre Derechos Humanos en Trabajo Social (NDHTS) y de Compromiso con los Derechos Humanos en Trabajo Social (CDHTS). Los resultados advierten la inferencia de la dimensión ventaja/utilidad en el componente vocacional del alumnado, así como un mayor peso de la dimensión aceptación de uno mismo y vida en cuanto a la resiliencia. Los conocimientos adquiridos en la formación profesional son superiores a los alcanzados en la vida cotidiana y el nivel de compromiso hacia los derechos humanos es medio-alto. Se observaron correlaciones directamente proporcionales entre las diferentes escalas y diferencias en función a las características sociodemográficas. Se considera necesario promover la inclusión de los DDHH en el currículum mediante una unidad de competencia específica.

Palabras clave: Derechos Humanos, formación, intervención, vocación y actitudes.

\begin{abstract}
Human Rights represent one of the fundamental principles in the international definition of Social Work. A quantitative research is proposed through a descriptivecorrelational design with the objective of analyzing the attitudes of the students of the Mexican Social Work degree towards human rights. A self-administered questionnaire was applied to 610 students from the Autonomous University of Nuevo León (UANL) and the University of Guadalajara (UdeG), selected through stratified random sampling. The instrument included: (1) a sociodemographic questionnaire, (2) the vocational component scale, (3) the scale of areas of intervention of Social Workers, (4) the Likert scale of resilience and (5) the Scale of Notions on Human Rights in Social Work (NDHTS) and of
\end{abstract}


Commitment to Human Rights in Social Work (CDHTS). The results show the inference of the advantage/utility dimension in the vocational component of the students, as well as a greater weight of the self-acceptance and life dimension in terms of resilience. The knowledge acquired in vocational training is higher than that achieved in daily life and the level of commitment to human rights is medium-high. Directly proportional correlations were observed between the different scales and differences based on sociodemographic characteristics. It is considered necessary to promote the inclusion of human rights in the curriculum through a specific unit of competence.

Keywords: Human Rights, training, intervention, vocation and attitude.

Sumario: 1. Introducción. 2. Contextualización. 3. Método. 4. Resultados. 5. Discusión. 6. Conclusiones.

\section{Introducción}

La noción de Derechos Humanos (DDHH) puede ser abordada desde un análisis lógico o desde una perspectiva ostentativa. Tomando como referencia esta última Guillén (2002) define los DDHH como derechos fundamentales (individuales y colectivos) de los seres humanos fruto de conquistas históricas que tienen y deben de ser reconocidos y respetados. Los cuales se caracterizan por ser universales, integrales e indivisibles, intransferibles, irrenunciables y generadores de derechos y deberes. De modo que diferencia tres generaciones de DDHH: Derechos civiles y políticos, Derechos económicos, sociales y culturales y Derechos de solidaridad. En este mismo sentido Camarena (2015) indica que los DDHH incluyen normas y relaciones de poder que se materializan a través de discursos, hechos e identidades. Así señala que son extrajurídicos (fenómeno multidimensional), están vinculados con los derechos económicos, sociales y culturales y por ende tienen una incidencia directa en las transformaciones políticas.

El enfoque en DDHH emerge como una alternativa el marco socio-jurídico hegemónico para la creación de políticas de atención, promoción y protección de los DDHH, así como 
de vigilancia y de exigibilidad de los mismos (Cubillos-Vega, 2018). De esta forma, como indican Mancinas, Zúñiga, Arroyo, Rodríguez-Otero y Támez (2017), el enfoque de DDHH se presenta como un paradigma alternativo a los tradicionales en las Ciencias Sociales y por ende en el Trabajo Social. Lo cual se constata a través de su inclusión en la definición que la Federación Internacional del Trabajo Social [FITS] (2104) expone sobre el Trabajo Social ${ }^{1}$, en la cual indica que los DDHH son uno de los principios fundamentales para la profesión, junto a la justicia social, la responsabilidad colectiva y el respeto a la diversidad. Asimismo, la FITS (2104) enfatiza señalando que:

La defensa y el apoyo a los derechos humanos y la justicia social son la motivación y la justificación para el trabajo social. La profesión del trabajo social reconoce que los derechos humanos tienen que coexistir con la responsabilidad colectiva. La idea de la responsabilidad colectiva destaca la realidad de que los derechos humanos individuales sólo se pueden alcanzar en el día a día si las personas asumen la responsabilidad de los demás y el medio ambiente, y la importancia de crear relaciones recíprocas dentro de las comunidades. Por lo tanto, un aspecto importante del trabajo social es abogar por los derechos de las personas en todos los niveles, y facilitar los resultados para que las personas asuman la responsabilidad por el bienestar del otro.

Es por ello que autores como Mellizo (2008) destacan que su vinculación radica en cuestiones históricas, ético-políticas y científicas, en lo que define como interdependientes en cuanto a la democracia, el desarrollo humano y la ciudadanía, en aras de la justicia social. De esta forma la identidad y la exclusión se presentan como categorías fundamentales en la interpretación de la realidad y por tanto en la acción profesional.

Diferentes autores del contexto español destacan la importancia que tiene el Trabajo Social en la defensa y promoción de los DDHH. Sin embargo, evidencian su escasa visibilización en los planes formativos de la mayoría de las universidades españolas; así como la necesidad de ampliar y/p profundizar en el contenido teórico y práctico, en cuanto al enfoque de DDHH y la ética del Trabajo Social, especialmente en lo referente al principio de autonomía (Cubillos-Vega 2018; Cubillos-Vega y Ferrán-Aranaz, 2019; Cubillos-Vega, Ferrán-Aranaz, Mercado y Pastor, 2017). Es por ello que Martínez (2016) cita las

\footnotetext{
${ }^{1}$ El trabajo social es una profesión basada en la práctica y una disciplina académica que promueve el cambio y el desarrollo social, la cohesión social, y el fortalecimiento y la liberación de las personas. Los principios de la justicia social, los derechos humanos, la responsabilidad colectiva y el respeto a la diversidad son fundamentales para el trabajo social. Respaldada por las teorías del trabajo social, las ciencias sociales, las humanidades y los conocimientos indígenas, el trabajo social involucra a las personas y las estructuras para hacer frente a desafíos de la vida y aumentar el bienestar.
} 
representaciones de los DDHH en torno al Trabajo Social como: (1) eje vertebrador de la profesión, (2) valores insertos en su conceptualización, (3) principios éticos profesionales, (4) teoría académica (dimensión epistemológica) y (5) referencia para la práctica profesional (dimensión ontológica). Asimismo, Vivero-Arriagada (2020) pone de manifiesto que, en contextos de conflictividad social en los que el neoliberalismo genera desigualdades poblacionales, la figura del Trabajo Social es fundamental para la promoción, la defensa y efectividad de los $\mathrm{DDHH}$ entre la población; por lo que la formación de los futuros profesionales para tales fines requiere de formación específica de los mismos. Es por ello que Løkke (2012) señala que la educación en DDHH se perfila como un medio de difusión del conocimiento e instrucción orientado al desarrollo de valores y competencias necesarias para la promoción, el respeto y la defensa de tales derechos. De esta forma se gesta la creación de un marco de cambio social orientado a la emancipación de los sujetos, el desarrollo comunitario y la transformación social (Hawkins y Knox, 2014); el cual toma especial importancia en la praxis de los trabajadores/as sociales en tanto en cuanto a la defensa de los derechos fundamentales de los sujetos y la acción comunitaria. Así la inclusión de este tipo de contenidos en los planes formativos de la licenciatura en Trabajo Social resulta determinante.

En este sentido, Cubillos-Vega (2019) indica que de 37 universidades españolas que imparten el grado en Trabajo Social el 54\% aluden a los DDHH y el 19\% imparten una materia específica. Asimismo, Reyes-Pérez, Hasse-Riquelme y Silva-Burgos (2020) señalan que, en 32 universidades de Chile, solamente el 31.2\% refieren explícitamente a la formación orientada a los DDHH en los planes formativos. En Costa Rica, Herrera (2014) también evidencia su escasa visibilidad y optatividad para el alumnado, así como la existencia de un claro posicionamiento iusnaturalista. En referencia al contexto mexicano, cabe destacar que en 2001 se celebró la conferencia Regional sobre Educación en Derechos Humanos (DDHH) en América Latina y el Caribe en Ciudad de México; en la cual, como señala Duarte-Hidalgo (2014), se exhortó a los Estados a incorporar la perspectiva de DDHH y las políticas de igualdad en los planes educativos. No obstante, en México no se advierten estudios que aborden y analicen su inclusión en los planes formativos de Trabajo Social. 
El análisis sobre las actitudes sobre DDHH en Trabajo Social, como señalan CubillosVega, Ferrán-Aranaz, Mercado y Pastor (2017), es escaso; no obstante, los autores mencionan las investigaciones de McFarland y Mathews (2005) sobre predictores actitudinales, el instrumento Human Rights Questionnarie (HQR) en sus diferentes versiones (Díaz-Veizades, Widaman, Little y Gibbs, 1995; Crowson, 2004; McFarland y Mathews, 2005). Asimismo, Cubillos-Vega, Ferrán-Aranaz, Mercado y Pastor (2017) citan otros estudios en los que incluyen las escalas Human Right Engagement in Social Work (HRESW) y Human Right Exposure in Social Work (HRXSW) sobre conocimiento y compromiso hacia los DDHH (McPherson y Abell, 2012; Chen, Tung, y Tang, 2015; Steen, Mann y Gryglewic, 2016; Šadić, McPherson, Villarreal-Otálora y Bašić, 2020). Ambas escalas validadas y traducidas al español por Cubillos-Vega, Ferrán-Aranaz y McPherson (2015) como la Escala de Nociones sobre Derechos Humanos en Trabajo Social (NDHTS) y la Escala de Compromiso con los Derechos Humanos en Trabajo Social (CDHTS). Asimismo, se encuentran otros estudios específicos sobre Trabajo Social que incluyen cuestionarios no validados (Sanz y Millán, 2019; Padrón, 2018). En este sentido los autores manifiestan que estos instrumentos, hasta el momento, son los únicos que "miden las dimensiones de la exposición a los derechos humanos (incluyendo la experiencia y la formación recibida) y el comportamiento de los estudiantes de Trabajo social, medido a través del compromiso hacia los derechos humanos, que incluye los componentes: adhesión, relevancia y práctica" (Cubillos-Vega, Ferrán-Aranaz, Mercado y Pastor, 2017, p. 1.053). A través del acervo expuesto se aprecia que en el contexto mexicano no existen investigaciones que aborden las actitudes sobre DDHH en Trabajo Social. Por otro lado, solamente una investigación en España analiza la relación entre tales actitudes y el componente vocacional del alumnado (Cubillos-Vega, Ferrán-Aranaz, Mercado y Pastor, 2017). Asimismo, no se aprecian estudios que tomen en consideración la preferencia del alumnado sobre los ámbitos de intervención de Trabajadores/as Sociales y el nivel de resiliencia de los mismos. Es por ello que se plantea una investigación cuantitativa con el objetivo de analizar las actitudes del alumnado de la licenciatura de Trabajo Social hacia los derechos humanos del alumnado en la Universidad Autónoma de Nuevo León (UANL) y la Universidad de Guadalajara (UdeG). Así como los siguientes objetivos específicos: (1) medir el componente vocacional del alumnado de Trabajo Social, 
(2) determinar los ámbitos de intervención de preferencia del alumnado, (3) constatar la resiliencia que poseen los estudiantes de Trabajo Social, (4) examinar las nociones y el compromiso hacia los DDHH que poseen los estudiantes, (5) identificar la relación existente entre componente vocacional, los ámbitos de intervención de preferencia, la resiliencia, las nociones y el compromiso hacia los DDHH del alumnado y (6) comparar los resultados en función al sexo, ámbito, semestre, creencias religiosas y universidad del alumnado de Trabajo Social.

\section{Contextualización}

En México se imparte la licenciatura de Trabajo Social en 31 universidades, de las cuales tomando en consideración las universidades públicas en los Estados de Nuevo León y Jalisco solamente se oferta en la UANL y en la UdeG. En el curso 2019-2020 en la Facultad de Trabajo Social de la UANL el número de matriculados fue de 1.700 alumnos/as en los 9 semestres, mientras que en la UdeG de 1.149 alumnos/as en los 8 semestres en que se articula.

Respecto a la UANL destaca que, en la web de la facultad, la presentación de la titulación hace referencia a los DDHH tanto en el enfoque (FTSyDH, 2020a) como en la Misión y visión de la entidad (FTSyDH, 2020b).

Enfoque. Se caracteriza por ser un programa científico-práctico, con un sustento teórico proporcionado por las Ciencias Sociales y Humanísticas [...] Los principios de los derechos humanos y la justicia social son fundamentales en este quehacer (FTSyDH, 2020a).

Misión. La FTSyDH es una dependencia académica de la UANL que tiene la misión de la formación de profesionales del Trabajo Social y Desarrollo Humano [...] mediante la utilización de teorías sobre el comportamiento humano y los sistemas sociales, el trabajo social interviene en los puntos en que las personas interactúan con su entorno. Los principios de los derechos humanos y la justicia social son fundamentales en este quehacer. 
En cuanto a la UdeG, en la presentación de la titulación se incluyen nociones a los DDHH en referencia a la descripción del perfil de egreso del alumnado (CUCSH, 2020); sin embargo en la misión y visión no se identifican referencias.

Perfil de egreso. Que el egresado de la Licenciatura en Trabajo Social será un profesional de las Ciencias Sociales con una sólida formación en el conocimiento y comprensión de la sociedad contemporánea, comprometido con el desarrollo de la democracia; promueve la participación, la ciudadanía, la justicia social y los derechos humanos [...] (CUCSH, 2020).

Por otro lado, se advierte que en la UANL no se incluyen unidades de aprendizaje (materias) específicas sobre DDHH, solamente se imparte una unidad obligatoria del área de formación básica vinculada al Derecho denominada Fundamentos de Derecho para el Trabajo Social.

Mientras que en el plan de estudios de la UdeG se incluye una unidad de aprendizaje específica sobre DDHH que es de carácter obligatorio del área de formación básica común denominada Derechos Humanos, la cual tiene una carga lectiva de 6 créditos (60 horas). Asimismo, incorpora una unidad obligatoria del área de formación básica vinculada al Derecho denominada Derecho Constitucional I.

\section{Método}

Se realizó una investigación cuantitativa mediante un diseño descriptivo-correlacional. La muestra total fue de 610 personas, compuesta por estudiantes de la licenciatura de Trabajo Social de la Universidad Autónoma de Nuevo León (UANL) y la Universidad de Guadalajara (UdeG). Se utilizó el criterio de oportunidad para seleccionar ambos contextos, debido a la disponibilidad de acceso a las universidades a través de los investigadores que participan en el estudio. Para la selección de la muestra se utilizó un muestreo aleatorio estratificado (margen de error 5\% y nivel de confianza 95\%) de cada uno de los semestres. 


\begin{tabular}{|c|c|c|c|c|c|c|c|c|}
\hline & & \multicolumn{2}{|c|}{ UANL } & \multicolumn{2}{|c|}{ UdeG } & \multicolumn{2}{|c|}{ Total } & \\
\hline & & $\mathrm{n}$ & $\%$ & $\mathrm{n}$ & $\%$ & $\mathrm{n}$ & $\%$ & \\
\hline \multirow[t]{2}{*}{ Sexo } & $\begin{array}{l}\text { Hombre } \\
\text { s }\end{array}$ & 21 & 6.4 & 25 & 8.9 & $\begin{array}{l}4 \\
6\end{array}$ & $\begin{array}{l}7 . \\
5\end{array}$ & \\
\hline & Mujeres & $\begin{array}{c}20 \\
7\end{array}$ & 93.6 & $\begin{array}{c}25 \\
7 \\
\end{array}$ & 91.1 & \multicolumn{2}{|c|}{564} & $\begin{array}{c}92 . \\
5\end{array}$ \\
\hline \multirow[b]{2}{*}{ Ámbito } & Rural & 32 & 9.8 & 26 & 9.2 & \multicolumn{2}{|l|}{58} & 9.5 \\
\hline & Urbano & $\begin{array}{c}29 \\
6\end{array}$ & 90.2 & $\begin{array}{c}25 \\
6\end{array}$ & 90.8 & \multicolumn{2}{|c|}{552} & $\begin{array}{c}90 . \\
5\end{array}$ \\
\hline \multirow{2}{*}{$\begin{array}{l}\text { Creencia } \\
\text { s } \\
\text { religiosas }\end{array}$} & $\mathrm{Si}$ & $\begin{array}{c}26 \\
6\end{array}$ & 81.1 & $\begin{array}{c}21 \\
5\end{array}$ & 76.2 & \multicolumn{2}{|c|}{481} & $\begin{array}{c}78 . \\
9\end{array}$ \\
\hline & No & 62 & 18.9 & 67 & 23.8 & \multicolumn{2}{|c|}{129} & $\begin{array}{c}21 . \\
1\end{array}$ \\
\hline \multirow{8}{*}{ Semestre } & 1 & 41 & 12.5 & 57 & 20.2 & \multicolumn{2}{|l|}{98} & $\begin{array}{c}16 . \\
1\end{array}$ \\
\hline & 2 & 41 & 12.5 & 28 & 9.9 & \multicolumn{2}{|l|}{69} & $\begin{array}{c}11 . \\
3\end{array}$ \\
\hline & 3 & 41 & 12.5 & 32 & 11.3 & \multicolumn{2}{|l|}{73} & $\begin{array}{c}12 . \\
0\end{array}$ \\
\hline & 4 & 41 & 12.5 & 35 & 12.4 & \multicolumn{2}{|l|}{76} & $\begin{array}{c}12 . \\
5\end{array}$ \\
\hline & 5 & 41 & 12.5 & 44 & 15.6 & \multicolumn{2}{|l|}{85} & $\begin{array}{c}13 . \\
9\end{array}$ \\
\hline & 6 & 41 & 12.5 & 29 & 10.3 & \multicolumn{2}{|l|}{70} & $\begin{array}{c}11 . \\
5\end{array}$ \\
\hline & 7 & 41 & 12.5 & 31 & 11.0 & \multicolumn{2}{|l|}{72} & $\begin{array}{c}11 . \\
8\end{array}$ \\
\hline & 8 & 41 & 12.5 & 26 & 9.2 & \multicolumn{2}{|l|}{67} & $\begin{array}{c}11 . \\
0\end{array}$ \\
\hline \multicolumn{2}{|c|}{ Total } & $\begin{array}{c}32 \\
8\end{array}$ & $\begin{array}{c}100 / 53 . \\
7\end{array}$ & $\begin{array}{c}28 \\
2\end{array}$ & $\begin{array}{c}100 / 46 . \\
3\end{array}$ & \multicolumn{2}{|c|}{610} & 100 \\
\hline
\end{tabular}

Tabla 1. Características sociodemográficas de la muestra.

Se aplicó un cuestionario autoadministrado compuesto por: (1) cuestiones sociodemográficas, (2) la escala de componente vocacional de Cubillos-Vega, FerránAranaz, Mercado y Pastor (2017), (3) la escala de ámbitos de intervención de Trabajadores/as Sociales de Viscarret, Ballestero, Idareta y Úriz (2016), (4) la escala Likert de resiliencia de Corchado, Díaz-Aguado y Martínez (2017), (5) la Escala de Nociones sobre Derechos Humanos en Trabajo Social (NDHTS) y (6) la Escala de Compromiso con los Derechos Humanos en Trabajo Social (CDHTS); ambas escalas traducidas y validadas por Cubillos-Vega, Ferrán-Aranaz y McPherson (2015). 
La escala de componente vocacional de Cubillos-Vega, Ferrán-Aranaz, Mercado y Pastor (2017) y la escala de ámbitos de intervención de Trabajadores/as Sociales de Viscarret, Ballestero, Idareta y Úriz (2016) están construidas en formato Likert con valores de 1 a 10, considerando el valor mínimo como totalmente en desacuerdo y el máximo como totalmente de acuerdo en cada uno de los enunciados. La escala de componente vocacional de Cubillos-Vega, Ferrán-Aranaz, Mercado y Pastor (2017) está conformada por tres dimensiones (Dimensión 1 vocacional/social, Dimensión 2 ventaja/utilidad y Dimensión 3 intermedia).

La escala de resiliencia de Corchado, Díaz-Aguado y Martínez (2017) está construida en formato Likert con valores de 1 a 7, considerando el valor mínimo como totalmente en desacuerdo y el máximo como totalmente de acuerdo en cada uno de los enunciados; incluye dos componentes (Dimensión 1 competencia personal y Dimensión 2 aceptación de uno mismo y de su vida). Finalmente, las escalas NDHTS y CDHTS están construidas en formato Likert con valores de 1 a 7 , considerando el valor mínimo como totalmente en desacuerdo y el máximo como totalmente de acuerdo en cada uno de los enunciados. La primera versa sobre conocimiento y la exposición hacia contenidos de Derechos Humanos e incluye dos dimensiones (Dimensión 1 nociones adquiridas en la formación profesional y Dimensión 2 nociones adquiridas en la vida cotidiana), mientras que la segunda mide el compromiso hacia los derechos humanos.

Tras el diseño del protocolo de investigación se presentó en cada universidad y se solicitaron los permisos necesarios. Se aplicó el cuestionario en cada universidad de forma voluntaria, anónima y en horario escolar a través de docentes previamente seleccionados. La aplicación de los instrumentos se realizó entre septiembre y diciembre de 2019 y tuvo una duración de 30 minutos. Tras la recolección de todos los instrumentos se codificaron en el paquete estadístico SPSS versión 22 y se invirtieron las respuestas de los ítems 15, 36 y 40 del cuestionario. Se calcularon los totales y frecuencia de las cuestiones sobre las características sociodemográficas, así como la media y desviación típica de cada escala. Posteriormente, se realizó la comparación de medias entre las variables universidad, sexo, ámbito, creencias religiosas y nivel de instrucción y la prueba paramétrica t-Student. En las variables cuyo nivel de significancia fue inferior a 0.05 se calculó la correlación bilateral 
de Rho de Spearman a nivel 0.01 y 0.05 . Asimismo, se analizaron las correlaciones bilaterales de Pearson a nivel 0.01 y 0.05 entre las escalas aplicadas.

Cabe destacar que la selección de las variables sociodemográficas objeto de análisis se realizó en función a las incluidas en el estudio de Cubillos-Vega, Ferrán-Aranaz, Mercado y Pastor (2017).

\section{Resultados}

Los resultados obtenidos a través de la Escala de componente vocacional (ver tabla 2) denotan que la elección de la licenciatura en Trabajo Social se concentró en las variables de interés problemáticas, políticas y bienestar social $\left(\mathrm{P} .1 ; \mathrm{x}^{2}=8.94 ; \sigma=1.64\right)$, ayuda a las personas necesitadas $\left(\mathrm{P} .2 ; \mathrm{x}^{2}=8.11: \mathrm{dt}=2.24\right)$ y trabajo con las personas $(\mathrm{P} .6 ; \mathrm{x}=8.65 ; \sigma$ =2.10). No obstante, la comparación entre las 3 dimensiones mediante el escalamiento multidimensional refleja que: (1) la Dimensión vocacional con mayor componente social (P.1, P.2 y P.6) presenta una puntuación alta $\left(\mathrm{x}^{2}=8.57 ; \sigma=1.42\right),(2)$ la Dimensión ventajautilidad (P.3, P.5, P. 8, P. 9 y P.10) media-baja $\left(\mathrm{x}^{2}=4.18: \mathrm{dt}=1.42\right)$ y (3) la Dimensión intermedia (P.4 y P7) media-alta $\left(\mathrm{x}^{2}=6.95 ; \sigma=2.25\right)$. Asimismo, se advierten puntuaciones homogéneas en ambas universidades respecto a la Dimensión vocacional con mayor componente social, mientras que en cuanto a la Dimensión ventaja-utilidad la UANL refleja puntuaciones mayores $\left(x^{2}=4.62 ; \sigma=2.14\right)$ respecto a la $U d e G\left(x^{2}=3.68 ; \sigma=1.77\right)$. En este sentido cabe destacar que tras realizar la puebla t-Student de comparación de medias, los resultados arrojaron niveles aceptables a excepción de la dimensión ventaja-utilidad; identificándose un nivel de significancia bilateral inferior a 0.05 (ver tabla 2). No obstante, tras realizar la prueba no paramétrica de Rho de Spearman se aprecia un coeficiente de correlación inversamente proporcional a nivel 0.01 (Coeficiente $=-0.223 ; \mathrm{p}=0.000$ ); por lo que se aceptan los resultados.

Las puntuaciones del alumnado respecto a la Escala de resiliencia reflejan que la aceptación de uno mismo y de su vida $\left(\mathrm{x}^{2}=5.95 ; \sigma=1.39\right)$ es ligeramente superior a la dimensión competencia personal $\left(x^{2}=5.65 ; \sigma=0.96\right)$. No obstante, como se observa en la 
tabla 2, se aprecian diferencias significativas entre ambas universidades. Así, aunque la dimensión aceptación de uno mismo es superior en sendas instituciones a la dimensión competencia personal, en la UANL se registran puntuaciones mayores; especialmente respecto a la segunda. Al respecto, cabe señalar que la prueba t-Student manifiesta niveles aceptables en cuanto sendas dimensiones (ver tabla 2).

En cuanto a la Escala de Nociones sobre Derechos Humanos en Trabajo Social (ver tabla 2), se aprecia que las puntuaciones tanto en la dimensión relativa a las nociones adquiridas en la formación profesional $\left(\mathrm{x}^{2}=5.03 ; \sigma=1.12\right)$, como en la dimensión de nociones adquiridas en la vida cotidiana $\left(\mathrm{x}^{2}=4.21 ; \sigma=0.94\right)$, son medio-altas en cuanto a la primera, medias respecto a la segunda y ligeramente superior la primera en ambas universidades. Asimismo, destaca que en la UdeG la dimensión nociones adquiridas en la formación profesional $\left(\mathrm{x}^{2}=5.26 ; \sigma=0.99\right)$ es superior a la de la UANL $\left(\mathrm{x}^{2}=4.83 ; \sigma=1.19\right)$; mientras que la dimensión de nociones adquiridas en la vida cotidiana es superior en la UANL $\left(\mathrm{x}^{2}=4.23 ; \sigma=1.01\right)$ respecto a la UdeG $\left(\mathrm{x}^{2}=4.23 ; \sigma==1.01\right)$. En este sentido cabe destacar que tras realizar la prueba t-Student de comparación de medias, los resultados arrojaron niveles aceptables a excepción de la dimensión nociones en la dimensión profesional en la cual el nivel de significancia bilateral es inferior a 0.05 (ver tabla 2). No obstante, tras realizar la prueba no paramétrica de Rho de Spearman se aprecia un coeficiente de correlación directamente proporcional a nivel 0.01 (Coeficiente $=0.190 ; \mathrm{p}=0.000$ ); por lo que se aceptan los resultados.

Respecto a la Escala de Compromiso con los Derechos Humanos en Trabajo Social (ver tabla 2), las puntuaciones describen un nivel de compromiso medio-alto $\left(\mathrm{x}^{2}=5.15 ; \sigma=0.79\right)$ y ligeramente superior en la $\operatorname{UdeG}\left(\mathrm{x}^{2}=5.23 ; \sigma=0.77\right)$ respecto a la UANL $\left(\mathrm{x}^{2}=5.10 ; \sigma=\right.$ 0.81); identificando un nivel aceptable en la puebla t-Student (ver tabla 2). 


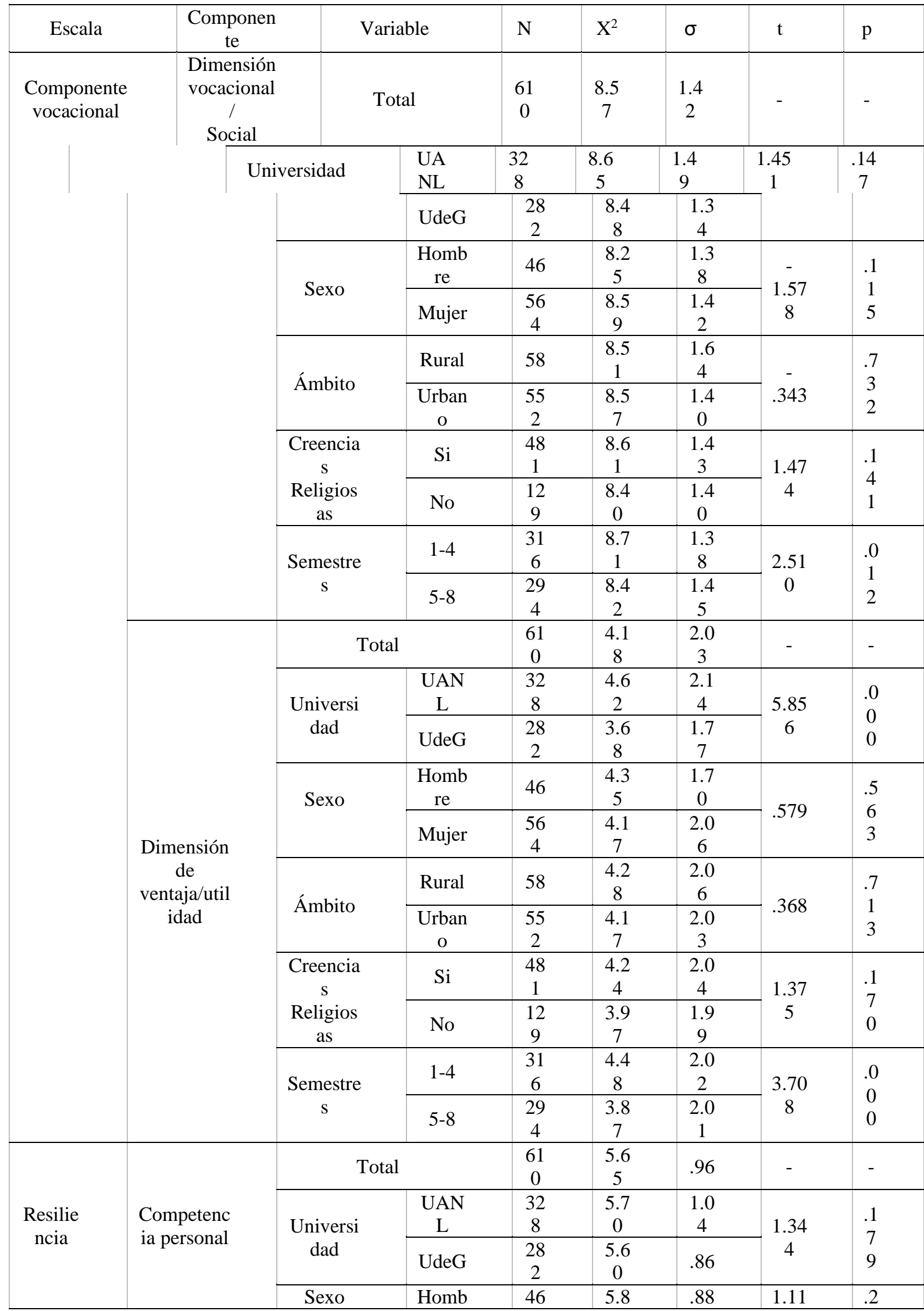




\begin{tabular}{|c|c|c|c|c|c|c|c|c|}
\hline & & & re & & 1 & & \multirow[t]{2}{*}{6} & \multirow{2}{*}{$\begin{array}{l}6 \\
5\end{array}$} \\
\hline & & & Mujer & $\begin{array}{c}56 \\
4\end{array}$ & $\begin{array}{c}5.6 \\
4\end{array}$ & .97 & & \\
\hline & & \multirow{2}{*}{ Ámbito } & Rural & 58 & $\begin{array}{c}5.3 \\
4\end{array}$ & $\begin{array}{c}1.6 \\
4\end{array}$ & \multirow{2}{*}{$\begin{array}{c}- \\
2.63 \\
7\end{array}$} & \multirow{2}{*}{$\begin{array}{l}.0 \\
0 \\
9\end{array}$} \\
\hline & & & $\begin{array}{c}\text { Urban } \\
\text { o }\end{array}$ & $\begin{array}{c}55 \\
2\end{array}$ & $\begin{array}{c}5.6 \\
9\end{array}$ & .85 & & \\
\hline & & \multirow{2}{*}{$\begin{array}{c}\text { Creencia } \\
\text { s } \\
\text { Religios } \\
\text { as } \\
\end{array}$} & $\mathrm{Si}$ & $\begin{array}{c}48 \\
1\end{array}$ & $\begin{array}{c}5.6 \\
9\end{array}$ & .91 & \multirow{2}{*}{$\begin{array}{c}1.92 \\
5\end{array}$} & \multirow{2}{*}{$\begin{array}{l}.0 \\
5 \\
5\end{array}$} \\
\hline & & & No & $\begin{array}{c}12 \\
9\end{array}$ & $\begin{array}{c}5.5 \\
1\end{array}$ & $\begin{array}{c}1.1 \\
3\end{array}$ & & \\
\hline & & \multirow{2}{*}{$\begin{array}{c}\text { Semestre } \\
\mathrm{s}\end{array}$} & $1-4$ & $\begin{array}{c}31 \\
6\end{array}$ & $\begin{array}{c}5.6 \\
5\end{array}$ & .91 & \multirow{2}{*}{$\begin{array}{c}- \\
.198\end{array}$} & \multirow{2}{*}{$\begin{array}{l}.8 \\
4 \\
3\end{array}$} \\
\hline & & & $5-8$ & $\begin{array}{c}29 \\
4 \\
\end{array}$ & $\begin{array}{c}5.6 \\
6 \\
\end{array}$ & $\begin{array}{c}1.0 \\
2 \\
\end{array}$ & & \\
\hline & \multirow{11}{*}{$\begin{array}{c}\text { Aceptación } \\
\text { de uno } \\
\text { mismo y } \\
\text { vida }\end{array}$} & \multicolumn{2}{|c|}{ Total } & $\begin{array}{c}61 \\
0\end{array}$ & $\begin{array}{c}5.9 \\
8\end{array}$ & $\begin{array}{c}1.1 \\
3\end{array}$ & - & - \\
\hline & & \multirow{2}{*}{$\begin{array}{c}\text { Universi } \\
\text { dad }\end{array}$} & $\begin{array}{c}\text { UAN } \\
\mathrm{L}\end{array}$ & $\begin{array}{c}32 \\
8\end{array}$ & $\begin{array}{c}6.0 \\
4\end{array}$ & $\begin{array}{c}1.2 \\
2\end{array}$ & \multirow{2}{*}{$\begin{array}{c}1.44 \\
7\end{array}$} & \multirow{2}{*}{$\begin{array}{l}.1 \\
4 \\
8\end{array}$} \\
\hline & & & UdeG & $\begin{array}{c}28 \\
2\end{array}$ & $\begin{array}{c}5.9 \\
0\end{array}$ & $\begin{array}{c}1.0 \\
3\end{array}$ & & \\
\hline & & \multirow[t]{2}{*}{ Sexo } & $\begin{array}{c}\text { Homb } \\
\text { re }\end{array}$ & 46 & $\begin{array}{c}6.0 \\
3 \\
\end{array}$ & $\begin{array}{c}1.2 \\
2 \\
\end{array}$ & \multirow{2}{*}{.343} & \multirow{2}{*}{$\begin{array}{l}.7 \\
3 \\
2\end{array}$} \\
\hline & & & Mujer & $\begin{array}{c}56 \\
4\end{array}$ & $\begin{array}{c}5.9 \\
7\end{array}$ & $\begin{array}{c}1.1 \\
3\end{array}$ & & \\
\hline & & \multirow{2}{*}{ Ámbito } & Rural & 58 & $\begin{array}{c}5.6 \\
0 \\
\end{array}$ & $\begin{array}{c}1.8 \\
4\end{array}$ & \multirow{2}{*}{$\begin{array}{c}- \\
2.66 \\
6\end{array}$} & \multirow{2}{*}{$\begin{array}{l}.0 \\
0 \\
8\end{array}$} \\
\hline & & & $\begin{array}{c}\text { Urban } \\
\mathrm{o}\end{array}$ & $\begin{array}{c}55 \\
2 \\
\end{array}$ & $\begin{array}{c}6.0 \\
2 \\
\end{array}$ & $\begin{array}{c}1.0 \\
3 \\
\end{array}$ & & \\
\hline & & \multirow{2}{*}{$\begin{array}{c}\text { Creencia } \\
\text { s } \\
\text { Religios } \\
\text { as } \\
\end{array}$} & $\mathrm{Si}$ & $\begin{array}{c}48 \\
1 \\
\end{array}$ & $\begin{array}{c}6.0 \\
7 \\
\end{array}$ & $\begin{array}{c}1.0 \\
4\end{array}$ & \multirow{2}{*}{$\begin{array}{c}3.83 \\
2\end{array}$} & \multirow{2}{*}{$\begin{array}{l}.0 \\
0 \\
0\end{array}$} \\
\hline & & & No & $\begin{array}{c}12 \\
9 \\
\end{array}$ & $\begin{array}{c}5.6 \\
4 \\
\end{array}$ & $\begin{array}{c}1.3 \\
7 \\
\end{array}$ & & \\
\hline & & \multirow{2}{*}{$\begin{array}{c}\text { Semestre } \\
\mathrm{s}\end{array}$} & $1-4$ & $\begin{array}{c}31 \\
6 \\
\end{array}$ & $\begin{array}{c}6.0 \\
1 \\
\end{array}$ & $\begin{array}{c}1.0 \\
4 \\
\end{array}$ & \multirow{2}{*}{.501} & \multirow{2}{*}{$\begin{array}{l}6 \\
1 \\
7\end{array}$} \\
\hline & & & $5-8$ & $\begin{array}{c}29 \\
4\end{array}$ & $\begin{array}{c}5.9 \\
5\end{array}$ & $\begin{array}{c}1.2 \\
2\end{array}$ & & \\
\hline \multirow{9}{*}{ DDHH } & \multirow{9}{*}{$\begin{array}{l}\text { Nociones } \\
\text { formación } \\
\text { profesional }\end{array}$} & \multicolumn{2}{|c|}{ Total } & $\begin{array}{c}61 \\
0\end{array}$ & $\begin{array}{c}5.0 \\
3\end{array}$ & $\begin{array}{c}1.1 \\
2\end{array}$ & - & - \\
\hline & & Universi & $\begin{array}{c}\text { UAN } \\
\mathrm{L}\end{array}$ & $\begin{array}{c}32 \\
8 \\
\end{array}$ & $\begin{array}{c}4.8 \\
3 \\
\end{array}$ & $\begin{array}{c}1.1 \\
9 \\
\end{array}$ & $\begin{array}{c}- \\
485\end{array}$ & $\begin{array}{l}.0 \\
0\end{array}$ \\
\hline & & dad & UdeG & $\begin{array}{c}28 \\
2\end{array}$ & $\begin{array}{c}5.2 \\
6\end{array}$ & .99 & $\begin{array}{c}4.0 J \\
5\end{array}$ & 0 \\
\hline & & Sexo & $\begin{array}{c}\text { Homb } \\
\text { re }\end{array}$ & 46 & $\begin{array}{c}4.8 \\
6 \\
\end{array}$ & .99 & - & .2 \\
\hline & & & Mujer & $\begin{array}{c}56 \\
4 \\
\end{array}$ & $\begin{array}{c}5.0 \\
4\end{array}$ & $\begin{array}{c}1.1 \\
3\end{array}$ & $\begin{array}{c}1.06 \\
8\end{array}$ & $\begin{array}{l}8 \\
6\end{array}$ \\
\hline & & Ám & Rural & 58 & $\begin{array}{c}4.8 \\
4 \\
\end{array}$ & $\begin{array}{c}1.5 \\
8 \\
\end{array}$ & - & $\begin{array}{l}.1 \\
8\end{array}$ \\
\hline & & Ambito & $\begin{array}{c}\text { Urban } \\
\mathrm{o}\end{array}$ & $\begin{array}{c}55 \\
2 \\
\end{array}$ & $\begin{array}{c}5.0 \\
5 \\
\end{array}$ & $\begin{array}{c}1.0 \\
6 \\
\end{array}$ & $\begin{array}{c}1.33 \\
3\end{array}$ & $\begin{array}{l}8 \\
3\end{array}$ \\
\hline & & $\begin{array}{c}\text { Creencia } \\
\mathrm{s}\end{array}$ & $\mathrm{Si}$ & $\begin{array}{c}48 \\
1 \\
\end{array}$ & $\begin{array}{c}5.0 \\
4\end{array}$ & $\begin{array}{c}1.1 \\
2\end{array}$ & 44 & .8 \\
\hline & & $\begin{array}{c}\text { Religios } \\
\text { as }\end{array}$ & No & $\begin{array}{c}12 \\
9 \\
\end{array}$ & $\begin{array}{c}5.0 \\
1 \\
\end{array}$ & $\begin{array}{c}1.1 \\
2\end{array}$ & .244 & 7 \\
\hline
\end{tabular}




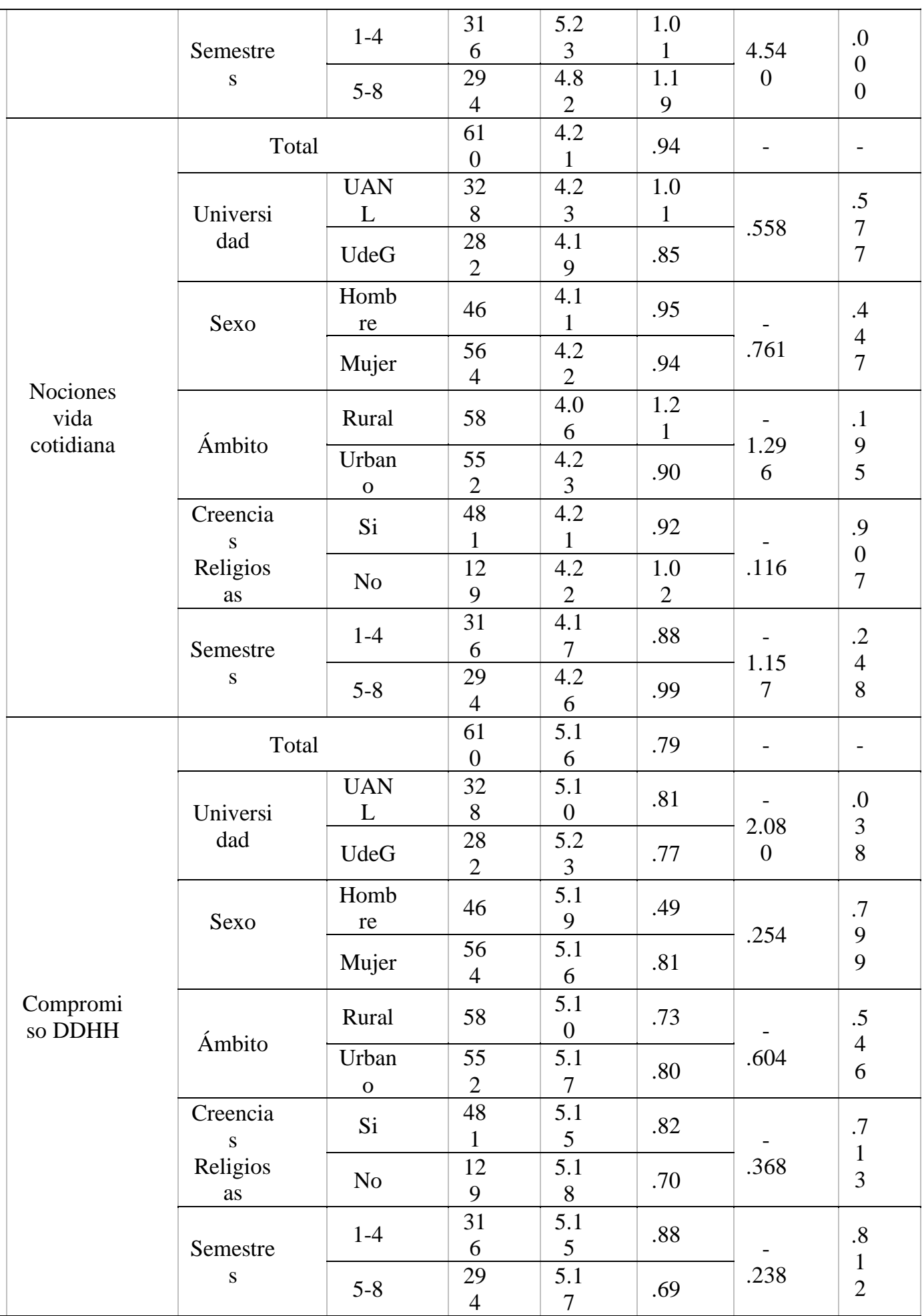

Tabla 2. Comparación de medias de las escalas en función a las características de la muestra

Nota: $\mathrm{N}=$ total; $\mathrm{x}^{2}=$ media; $\sigma=$ desviación estándar; $\mathrm{t}=$ valor $\mathrm{t}-$ Student; $\mathrm{p}=$ significancia bilateral. 
En cuanto a las variables analizadas (sexo, ámbito, creencias religiosas y nivel de instrucción), a través del análisis de comparación de medias se observa que, en referencia a la dimensión vocacional/social, las puntuaciones son ligeramente superiores en mujeres, en alumnado de ámbito urbano, con creencias religiosas y de los primeros semestres; identificando un nivel aceptable en la puebla t-Student respecto a todas las variables (ver tabla 2).

En función a la dimensión ventaja-utilidad se identifican medias superiores en hombres, participantes de entornos rurales, que manifiestan creencias religiosas y con un menor nivel de instrucción. Asimismo, se advierten en la comparación de medias niveles aceptables a excepción de la variable relativa al semestre (ver tabla 2). Tras realizar la prueba no paramétrica de Rho de Spearman se aprecia un coeficiente de correlación inversamente proporcional a nivel 0.01 (Coeficiente=-0.149; $\mathrm{p}=0.000$ ); por lo que se aceptan los resultados.

La competencia personal (resiliencia) es superior en mujeres, en alumnado de ámbito urbano, con creencias religiosas y de semestres más avanzados; mientras que en la dimensión de resiliencia de aceptación de uno mismo y de la vida los valores son mayores en hombres, en el ámbito urbano, participantes con creencias religiosas y con un menor nivel de instrucción. En este sentido cabe destacar que se identificó un nivel aceptable en la prueba t-Student respecto a todas las variables, a excepción de las creencias religiosas y la aceptación de uno mismo (ver tabla 2). Sin embargo, se advierte una correlación bilateral Rho de Spearman a nivel 0.01 (Coeficiente=-0.154; $\mathrm{p}=0.000$ ); por lo que se aceptan los resultados.

Por otro lado, en cuanto a las nociones de DDHH adquiridas a través de la formación, se registran medias superiores en mujeres, alumnado de ámbito urbano, con creencias religiosas y con menor nivel de instrucción; mientras que la implicación de las experiencias de la vida cotidiana en los DDHH, es superior en mujeres, alumnado de ámbito urbano, que no manifiesta creencias religiosas y de semestres más avanzados. Asimismo, se advierten niveles de significancia aceptables respecto a todas las variables, a excepción del nivel de instrucción y las nociones a través de acciones formativas (ver tabla 
2). Sin embargo, se advierte una correlación bilateral Rho de Spearman a nivel 0.01 (Coeficiente $=-0.175 ; \mathrm{p}=0.000)$; por lo que se aceptan los resultados.

En cuanto al nivel de compromiso hacia los DDHH se advierten valores superiores en hombres, alumnado de ámbito rural, participantes que no poseen creencias religiosas y con un nivel de instrucción superior; identificando un nivel aceptable en la prueba t-Student respecto a todas las variables (ver tabla 2).

Por otro lado, en la Escala de ámbitos de intervención de Trabajadores/as Sociales, se aprecia que las áreas con mayores puntuaciones son infancia, juventud, educación y familia $\left(x^{2}=8.46 ; \sigma=2.21\right)$, problemas conductuales e intervención familiar $\left(x^{2}=8.40 ; \mathrm{dt}=2.26\right) \mathrm{y}$ mujer $\left(\mathrm{x}^{2}=8.35 ; \sigma=2.22\right)$; mientras que las de menor interés se relacionan con las cuestiones burocráticas, el ámbito de inclusión (migración, racismo y minorías), adicciones, delincuencia y la intervención comunitaria. Asimismo, destaca que las puntuaciones entre ambas universidades son homogéneas, aunque entre el alumnado de la UANL superior respecto a las áreas de: adicciones, académica, gestión de Servicios Sociales, burocrática y diagnóstico y evaluación de la intervención (ver tabla 3).

A través del análisis correlacional de Pearson se advierte que existe una relación directamente proporcional entre casi todas las escalas aplicadas con nivel de significancia 0.01 a excepción de la dimensión aceptación de uno mismo y de su vida de la escala de resiliencia con respecto al grupo 3 (intermedio) de la escala de componente vocacional, entre las cuales se produce una correlación directamente proporcional a nivel 0.05.

En este sentido destaca que no se evidencian correlaciones, pero si una relación inversa entre el compromiso hacia los DDHH y el grupo 2 (ventaja-utilidad) de la escala de componente vocacional; así como respecto a las nociones de los DDHH a través de la formación y el componente vocacional ventaja-utilidad y también entre la dimensión aceptación (resiliencia) y el componente vocacional intermedio; resultando una relación directamente proporcional entre las mismas (ver tabla 4). 


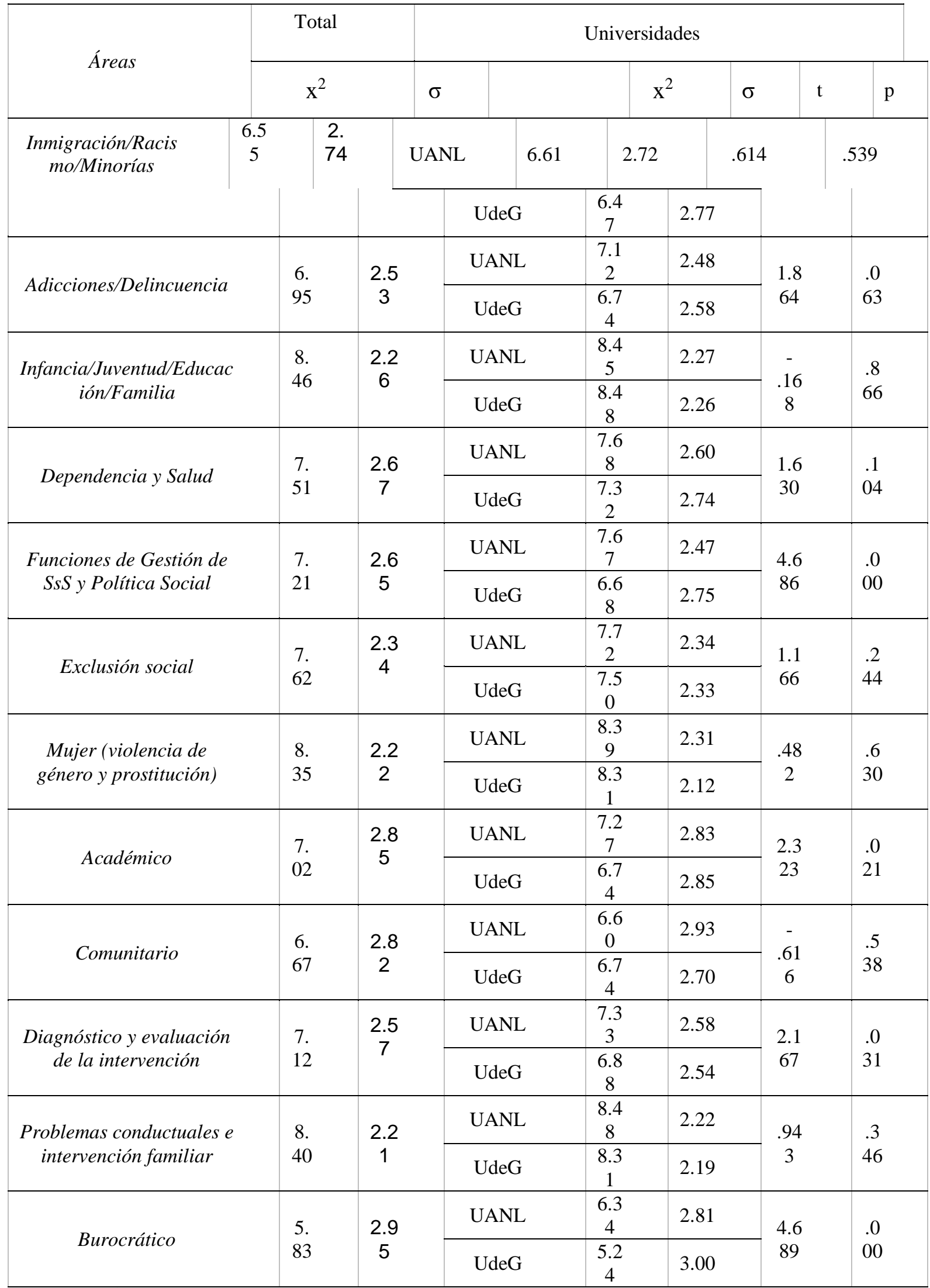

Tabla 3. Ámbitos de intervención de Trabajo Social según universidad. 
Nota: $\mathrm{N}=$ total; $\mathrm{x}^{2}=$ media; $\sigma=$ desviación estándar; $\mathrm{t}=$ valor $\mathrm{t}-\mathrm{Student} ; \mathrm{p}=$ significancia bilateral 


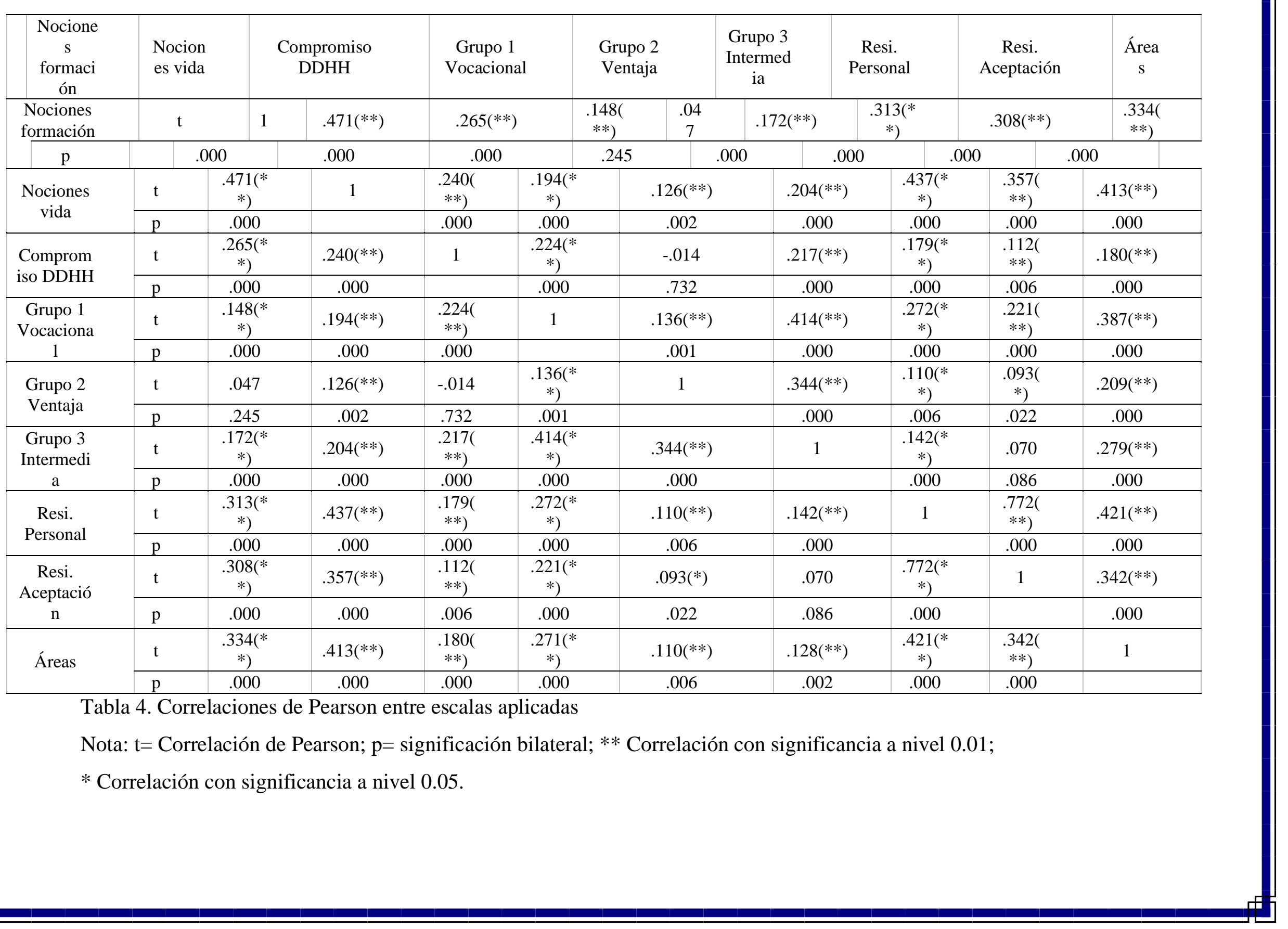




\section{Discusión}

Comparando los resultados obtenidos en la UANL y la UdeG con los presentados por Cubillos-Vega, Ferrán-Aranaz, Mercado y Pastor (2017) en las universidades españolas de UCM, UCLM y UM y Vega (2018) en la UCM se observa que: (1) en la escala de componente vocacional los valores de la Dimensión vocacional/social ( $\mathrm{x}^{2}$ México=8.575; $x^{2}$ España $=7.83$ ) son superiores a los valores de la Dimensión ventaja/utilidad y la Dimensión intermedia son superiores entre el alumnado mexicano respecto al español; especialmente en cuanto a las dimensiones dimensión intermedia $\left(x^{2}\right.$ México $\left.=6.95 ; x^{2} E s p a n ̃ a=4.03\right) \quad y \quad$ la dimensión ventaja/utilidad $\left(x^{2}\right.$ México $=4.18 ; x^{2}$ España $\left.=0.82\right)$. Respecto a los conocimientos y la exposición hacia contenidos de Derechos Humanos la Dimensión conocimientos adquiridos en la formación profesional refleja una ligera mayor presencia entre el alumnado español $\left(x^{2}=5.39\right)$ que en el mexicano $\left(\mathrm{x}^{2}=5.03\right)$, mientras que en la dimensión nociones adquiridas en la vida cotidiana el alumnado de México manifiesta un valor superior $\left(\mathrm{x}^{2}=4.21\right)$ respecto al español $\left(\mathrm{x}^{2}=3.88\right)$. Finalmente destaca que las puntuaciones del compromiso hacia los derechos humanos son superiores en el estudio realizado en España $\left(x^{2}=6.09\right)$ que en el de México $\left(x^{2}=5.16\right)$. Por otro lado, en referencia al estudio de Cubillos-Vega y FerránAranaz (2019) con alumnado de la UCM (España), se aprecian niveles inferiores en la muestra respecto a los indicados por los autores en cuanto a las NDHTS $\left(x^{2}=4.64\right)$ y el CDHTS $\left(x^{2}=6.21\right)$. Asimismo, en los estudios de Sanz y Millán (2019) y Padrón (2018) se identifican nivel de conocimiento menores.

Los estudios de Cubillos-Vega, Ferrán-Aranaz, Mercado y Pastor (2017), Cubillos-Vega y Ferrán-Aranaz (2019), McPerson y Abell (2012), McFarland, Mathews (2005) y Šadić, McPherson, Villarreal-Otálora y Bašić (2020), McFarland y Mathews (2005), Sanz y Millán (2019) y Padrón (2018) no incluyen la escala de resiliencia de Corchado, DíazAguado y Martínez (2017) y la escala de los ámbitos de intervención de Trabajadores/as Sociales de Viscarret, Ballestero, Idareta y Úriz (2016). Es por ello que en referencia a este criterio no es posible realizar una discusión con la literatura científica. 
Asimismo, solamente aborda un análisis correlacional entre las escalas NDHTS y CDHTS, las cuales describen como directamente proporcionales; aspecto también evidenciado por McPerson y Abell (2012), McFarland y Mathews (2005) y Šadić, McPherson, VillarrealOtálora y Bašić (2020). De esta forma la presente investigación evidencia la existencia de correlaciones entre todas las escalas y dimensiones a excepción de la dimensión compromiso hacia los DDHH y el grupo 2 (ventaja-utilidad) de la escala de componente vocacional, las nociones de los DDHH a través de la formación y el componente vocacional ventaja-utilidad y también entre la dimensión aceptación (resiliencia) y el componente vocacional intermedio.

Por otro lado, al igual que en el estudio de McFarland y Mathews (2005), se advierten diferencias significativas en cuanto a la dimensión nociones adquiridas en formación profesional de la escala NDHTS respecto al sexo, la edad y las creencias religiosas del alumnado, pero también en cuanto al ámbito (rural-urbano) y el nivel de instrucción. En este sentido, destaca que en la UdeG se imparte formación específica sobre DDHH y el alumnado registra puntuaciones notoriamente superiores; lo cual en el estudio de CubillosVega, Ferrán-Aranaz y McPherson (2015) no se demuestra. A pesar de identificar diferencia de medias en respecto a las características sociodemográficas de la mue stra en la dimensión nociones adquiridas en la vida cotidiana de la escala NDHTS y en la escala CDHTS, al igual que en el estudio de Cubillos-Vega, Ferrán-Aranaz y McPherson (2015), no se producen relaciones estadísticas significativas ya que las medias son semejantes. Por otro lado, en el estudio de Sanz y Millán (2019) se aprecian diferencia respecto al conocimiento en función del sexo; sin embargo, al no aplicarse el mismo instrumento, no es posible comparar los resultados. Asimismo, en las investigaciones de McPerson y Abell (2012), Cubillos-Vega, Ferrán-Aranaz y McPherson (2015) y Šadić, McPherson, Villarreal-Otálora y Bašić (2020) no es posible realizar una comparación al tratarse de validaciones de escalas que no incluyen análisis de comparación de medias. Por otro lado, en referencia al estudio de Cubillos-Vega y Ferrán-Aranaz (2019) con alumnado de la UCM (España) tampoco incluyen análisis de comparación de medias. 
Finalmente, los resultados de la presente investigación revelan que, además de identificar las diferencias significativas en cuanto a las variables descritas (sexo, universidad, creencias religiosas, ámbito geográfico y nivel de instrucción) y la dimensión nociones adquiridas en formación profesional de la escala NDHTS, también se producen diferencias significativas entre la dimensión ventaja-utilidad, la universidad, las creencias religiosas y el nivel de instrucción; así como también entre la dimensión aceptación de uno mismo de la escala de resiliencia y todas las variables analizadas. No obstante, es importante destacar que los resultados revelan que además de producirse correlaciones entre la escala de las NDHTS, el CDHTS (McFarland y Mathews, 2005), también se aprecia entre ambas escalas y todas las dimensiones de las escalas del componente vocacional, la resiliencia y las preferencias sobre los ámbitos de intervención de Trabajo Social.

\section{Conclusiones}

La disciplina del Trabajo Social interviene en escenarios sociales complejos, lo cual demanda una sólida formación académica que posibilite la comprensión y el análisis de las problemáticas sociales existentes en la sociedad contemporánea y su intervención, donde la vulneración a los derechos humanos es una constante.

Los futuros profesionales del Trabajo Social deben poseer lo conocimientos, habilidades y destrezas, así como las competencias necesarias para enfrentarse a escenarios complejos de intervención, donde promoverán en sus intervenciones los principios de justicia social, derechos humanos, responsabilidad colectiva y respeto a la diversidad. De ahí la importancia de que las escuelas formadoras contemplen en sus planes de estudio materias y contenidos que posibiliten una mayor comprensión del enfoque de derechos humanos en el estudiantado.

Los resultados obtenidos en la investigación permiten comparar los conocimientos que poseen las y los estudiantes de la carrera de Trabajo Social de dos universidades públicas de México en materia de DDHH, así como su nivel de compromiso y vocación, y poderlos contrastar con estudios previos realizados con estudiantes de universidades en España. Se evidencia la existencia de correlaciones entre todas las escalas, lo cual demuestra que a 
medida que los estudiantes tienen mayores conocimientos respecto a los DDHH, mayor es su compromiso en la promoción y defensa de ellos, es decir, hay una mayor implicación del estudiantado en aplicar el enfoque de derechos humanos como herramienta de intervención en la realidad social, lo cual impactará de manera favorable su futuro ejercicio profesional.

Puesto que actualmente un número significativo de escuelas formadoras se encuentran modificando sus planes de estudio, se considera pertinente ampliar el estudio a otras escuelas en México a fin de contar con una visión más global de la situación que guarda la formación académica de los futuros profesionales del Trabajo Social en materia de DDHH, y estar en posibilidades de elaborar recomendaciones. De esta forma el análisis global favorecería la incorporación de la educación en DDHH y la difusión del conocimiento e instrucción orientado al desarrollo de valores y competencias necesarias para la promoción, el respeto y la defensa de tales derechos. Permitiendo así dotar al alumnado de estrategias destinadas a la emancipación de los sujetos, el desarrollo comunitario y la transformación social.

Asimismo, sería conveniente tomar en consideración otras variables en el análisis como la participación en organizaciones civiles y organizaciones no gubernamentales (ONG) por parte del alumnado, el tipo de religión que profesan, las actitudes hacia el género y la diversidad sexual o el ámbito geográfico (rural/ urbano) entre otras variables.

\section{Referencias bibliográficas}

CAMARENA, C. S. (2015). La influencia de las clasificaciones de derechos humanos en la exigibilidad de los derechos económicos, sociales y culturales. Ciencia Jurídica, (7), 123-139.

Centro Universitario de Ciencias Sociales y Humanidades de la Universidad de Guadalajara [CUCSH] (2020). Presentación Licenciatura en Trabajo Social. Perfil de 
egreso.

Disponible

en

http://www.cucsh.udg.mx/perfilegreso/licenciatura_en_trabajo_social

CHEN, H.; TUNG, Y. y TANG, I. (2015). Teaching about human rights in a social work undergraduate curriculum: The Taiwan experience. British Journal of Social Work, 45(8), 2335-2350.

CORCHADO, A. I, DÍAZ-AGUADO, M. J., y MARTÍNEZ, R. (2017). Resiliencia en adolescentes expuestos a condiciones vitales de riesgo. Cuadernos de trabajo social, 30(2), 477-486.

CROWSON, H. M. (2004). Human rights attitudes: Dimensionality and psychological correlates. Ethics \& Behavior, 14(3), 235-253.

CUBILLOS-VEGA, C. (2018). La inclusión de los derechos humanos en la agenda del trabajo social: una aproximación a la educación en derechos humanos (EDH) en la formación académica (Tesis Doctoral). Madrid: Universidad Complutense de Madrid. Disponible en http://repositorio.conicyt.cl/bitstream/handle/10533/236388/Tesis_Carla\%20CubillosVega _2018_formato $\% 20$ libre.pdf?sequence $=1$

CUBILlOS-VEGA, C. (2019). La formación en derechos humanos en el Trabajo Social. Evolución, propuestas y retos. Comunitania, (17), 35-58.

CUBILLOS-VEGA， C. y FERRÁN-ARANAZ， M. (2019). Los efectos de una intervención educativa en materia de derechos humanos con alumnado de Trabajo Social: la valoración de las nociones y el compromiso. Cuadernos de Trabajo Social, 32(2), 381395. 
CUBILlOS-VEGA, C.; FERRAN-ARANAZ, M. y MCPHERSON, J. (2019). Bringing human rights to social work: Validating culturally appropriate instruments to measure rights-based practice in Spain. International Social Work, 62(5), 1343-1357.

CUBILlos-VEGA， C.; FERRÁN-ARANAZ， M.; MERCADO-GARCÍA， E. y PASTOR-SELLER, E. (2017). Nociones y compromiso con los derechos humanos en estudiantes de Trabajo Social en España. Revista mexicana de investigación educativa, 22(75), 1047-1075.

DIAZ-VEIZADES, J.; WIDAMAN, K.; LITTLE, T. y GIBBS, K. (1995). The measurement and structure of human rights attitudes. Journal of Social Psychology, 135(1), 313-328.

DUARTE-HIDALGO, C. (2014). La formación en Derechos Humanos como parte del proyecto ético político del Trabajo Social. Serviço Social \& Sociedade, (119), 482-507.

Facultad de Trabajo Social y Desarrollo Humano de la UANL [FTSyDH] (2020a). Presentación de la titulación. Disponible en http://ftsydh.uanl.mx/index.php/licenciatura/ Facultad de Trabajo Social y Desarrollo Humano de la UANL [FTSyDH] (2020b). Misión y visión. Disponible en http://ftsydh.uanl.mx/index.php/principal-3/mision-y-vision/

Federación Internacional del Trabajo Social [FITS] (2104). Definición global del Trabajo Social. Disponible en https://www.ifsw.org/what-is-social-work/global-definition-ofsocial-work/definicion-global-del-trabajo-social/

GUILLÉN, N. M. (2002). Derechos laborales en el marco de la globalización económica. Reflexiones, 81(1), 8-24.

LØKKE, М. (2012). The Human Rights Education Toolbox. A Practitioner's Guide to Planning and Managing Human Rights Education. Denmark: The Danish Institute for Human Rights. 
HAWKINS, C. y KNOX, K. (2014). Educating for international social work: Human rights leadership. International Social Work, 57(3), 248-257.

HERRERA, O. (2014). Trabajo Social: Formación académica y Derechos Humanos (Tesis de Licenciatura). San José: Universidad de Costa Rica. Disponible en https://www.academia.edu/35100228/Trabajo_Social_Formaci\%C3\%B3n_acad\%C3\%A9 $\underline{\text { mica_y_Derechos_Humanos }}$

MANCINAS, S. E.; ZÚÑIGA, M.; ARROYO, C.; RODRÍGUEZ-OTERO, L. M. y TÁMEZ, B. M. (2017). Teoría y modelos de Intervención en Trabajo Social. Fundamentos básicos y crítica I. Monterrey: Red Pública y UANL.

MARTínEZ, E. (2016). Derechos Humanos y Trabajo Social. Análisis de sus potencialidades en los proyectos de Cooperación Internacional para el Desarrollo (Tesis de Licenciatura). Barcelona: Universidad de Barcelona. Disponible en http://diposit.ub.edu/dspace/bitstream/2445/100714/1/Eloi_Mart\%C3\%ADnez_Avil\%C3\% A9s_TFG.pdf

McFARLAND, S. y MATHEWS, M. (2005). Who cares about human rights? Political Psychology, 26(3), 365-385.

McPHERSON, J. y ABELL, N. (2012). Human rights engagement and exposure new scales to challenge social work education. Research on Social Work Practice, 22(6), 704713.

MELLIZO, W.H. (2008). Trabajo social, Derechos Humanos y cuestión social: una praxis éticopolítica en tiempo de globalización. Didáctica, 2, 161-179.

PADRÓN, T. (2018). Trabajo Social y Derechos Humanos. Influencia de la formación en las nociones sobre Derechos Humanos del alumnado de Trabajo Social (Tesis de Licenciatura). Santa Cruz de Tenerife: Universidad de La Laguna. Disponible en https://riull.ull.es/xmlui/handle/915/9369 
REYES-PÉREZ, L. S., HASSE-RIQUELME, V. S., y SILVA-BURGOS, L. M. (2020). Educación en Derechos Humanos para el Trabajo Social en Chile: una mirada desde los estándares internacionales. PROSPECTIVA. Revista de Trabajo Social e Intervención Social, (30), 259-281.

ŠADIĆ, S.; McPHERSON, J.; VILLARREAL-OTÁlORA, T. y BAŠIĆ, S. (2020). Rights-based social work in Bosnia and Herzegovina: Validating tools for education and practice. International Social Work, 63(3). https://doi.org/10.1177/0020872820912310

SANZ, S. Á., y MILLÁN, M. J. (2019). Conocimiento sobre los Derechos Humanos del alumnado de Trabajo Social de la Universidad de La Laguna (Tesis de Licenciatura). Santa Cruz de Tenerife: Universidad de La Laguna. Disponible en https://riull.ull.es/xmlui/bitstream/handle/915/12236/Conocimiento\%20sobre\%2010s\%20D erechos $\% 20$ Humanos $\% 20 \mathrm{del} \% 20$ alumnado\%20de\%20Trabajo\%20Social\%20de $\% 201 \mathrm{a} \% 2$ 0Universidad\%20de\%20La\%20Laguna.pdf?sequence $=1$

Steen, J.; Mann, M. y Gryglewicz, K. (2016). The human rights philosophy: support and opposition among undergraduate Social Work students. Journal of Teaching in Social Work, 36(5), 446-459.

VISCARRET, J. J., BALLESTERO, A., IDARETA, F., y ÚRIZ, M. J. (2016). Tipologías actuales de los trabajadores sociales en España. Cuadernos de Trabajo Social, 29(2), 239.

VIVERO-ARRIAGADA, L.A. (2020). Condiciones para una Neo-Reconceptualización del Trabajo Social en Chile, Latinoamérica y el Caribe. Prospectiva. Revista de Trabajo Social e Intervención Social, (29), 193-212. 